COMMUNICATIONS IN

ANALYSIS AND GEOMETRY

Volume 12, Number 1, 321-343, 2004

\title{
The Futaki Invariant and the Mabuchi Energy of a Complete Intersection
}

\author{
D. H. Phong and Jacob Sturm
}

\section{Introduction.}

Let $M$ be a compact complex Kähler manifold. If $c_{1}(M)=0$ or if $c_{1}(M)<0$, then it is known by the work of Yau [Y78] and Yau, Aubin [Y78], [A78] that $M$ has a Kähler-Einstein metric. If $c_{1}(M)>0$, then there are obstructions to the existence of such a metric, and here the guiding conjecture is that formulated by Yau in [Y93], which says that $M$ has a Kähler-Einstein metric if and only if $M$ is stable in the sense of geometric invariant theory.

An important obstruction to the existence of Kähler-Einstein metric is the invariant of Futaki [F83], which is a map $F: \eta(M) \rightarrow \mathbf{C}$ with the following properties: $F$ is a Lie algebra character on the space $\eta(M)$ of holomorphic vector fields, which depends only on the cohomology class $[\omega] \in$ $H^{2}(M)$. The vanishing of $F$ is a necessary condition for the existence of a Kähler-Einstein metric on $M$. However, it is not a sufficient condition: in [T97], Tian gives an example of a manifold with $\eta(M)=0$ (so that the Futaki invariant vanishes trivially) with the property that $M$ has no KählerEinstein metric.

In [DT], a generalized Futaki invariant is defined for Q-Fano varieties, which are certain singular varieties arising naturally as degenerations of smooth Fano manifolds. In [T97], a manifold $M$ is defined to be K-stable if the Futaki invariant of every non-trivial Q-Fano degeneration of $M$ has positive real part. It is proved there that if $M$ has a Kähler-Einstein metric, then $M$ is $\mathrm{K}$-stable, and the converse is conjectured to be true as well.

In general, it is rather difficult to check the K-stability of a given manifold. Recent progress has been made by $\mathrm{Lu}$ [Lu01] in the case where $M$ is a hypersurface: Using a delicate analytic argument, he provides, in the case

\footnotetext{
${ }^{1}$ Research supported in part by the National Science Foundation under grants DMS-02-45371 and DMS-01-00410.
} 
where $M$ is a hypersurface, an explicit formula for the Futaki invariant of a degeneration of $M$. This represents a non-linear generalization in the special case of hypersurfaces of Lu's earlier formula [Lu99] for the Futaki invariant of a normal complete intersection.

In this paper we shall study the Futaki invariant and the Mabuchi Kenergy using the Deligne pairing technique. The Deligne pairing technique was introduced by Zhang [Z] and developed in [PS1] and [PS2]. Our main result is a formula (Theorem 6) for the Mabuchi energy on the orbits of $S L(N+1)$ which provides a non-linear version of Lu's Futaki invariant formula for a complete intersection. A similar formula is also established for the Aubin-Yau functional. We also give a simple characterization of the generalized Futaki invariant and a new construction of the generalized Futaki character as well. A basic idea in our approach is to associate to each smooth projective variety $M$ the one-dimensional vector space

$$
\mathcal{F}=\left\langle K^{-1}, \cdots, K^{-1}\right\rangle
$$

Here $K$ is the canonical bundle of $M,\langle\cdots\rangle$ is the Deligne intersection pairing, and $K^{-1}$ occurs $n+1$ times in the pairing. The point is that this construction is completely canonical, and hence the action of $\eta(M)$ on $M$ lifts to a group action on $\mathcal{F}$, which turns out to be precisely the Futaki character. A slightly modified version works in the Q-Fano case as well.

A more detailed description of the results and organization of the paper is as follows. Section 2 is devoted to establishing the simple characterization of the Futaki invariant: $F(X)$ is the eigenvalue of the infinitesimal action of $X$ on Chow $(M)$, the Chow point of $M$. Moreover, the Futaki character $\hat{F}(X)$ is the eigenvalue of the action of $\operatorname{Aut}(M)$ on Chow $(M)$ (see Theorem 1 and its corollary, stated in $\S 2.1$ and proved in $\S 2.4)$. The background needed on Deligne pairings is provided in $\S 2.2$. Energy functionals in Kähler geometry originate from many sources. A unifying theme is that of Bott-Chern secondary classes (see e.g. [Do], [FMS], [T00] and references therein). In this paper, we rely on another unifying theme discussed in $\S 2.3$, namely energy functionals as variations of metrics in suitable Deligne pairings (Theorem 2), e.g.

$$
\mathcal{M}_{h e^{-\phi}}=\mathcal{M}_{h} \otimes O\left(\nu_{\omega}(\phi)\right)
$$

Next, in Section 3, we show how one can use this characterization to give a new proof of Lu's formula [Lu99] on the generalized Futaki invariant of a complete intersection (Theorem 3). Section 4 begins with Theorem 4, which provides a general "adjunction formula with metrics". This theorem 
is closely related to a corresponding result of Lu, although the formulation in [Lu99] is rather different. Finally, we apply the adjunction formula with metrics in Theorems 5 and 6 to prove the desired formulas for the Aubin-Yau and the Mabuchi energy functionals for complete intersections. As stated earlier, these can be viewed as a non-linear version of Lu's formula for the Futaki invariant of complete intersections. They can also be viewed as a generalization to the case of complete intersections of formulas for hypersurfaces obtained earlier in [T94] and [PS1] for the Mabuchi functional, and in [Z] and [PS1] for the Aubin-Yau functional: in particular, we show that the K-energy of a complete intersection may be expressed as a degenerate norm on the space of defining polynomials.

\section{Lifting the Futaki character.}

\subsection{Statement.}

Let $M$ be an irreducible normal projective variety and $M_{r e g} \subseteq M$ the open subset consisting of all smooth points. We say $M$ is Q-Fano if there exists $k>0$ and a very ample line bundle $L$ on $M$ such that $\left.L\right|_{M_{r e g}}=K_{M_{r e g}}^{-k}$. A Kähler form $\omega$ on $M_{\text {reg }}$ is admissible if there exists $\phi_{L^{m}}: M \hookrightarrow \mathbf{P}^{N}$, an embedding of $M$ defined by some power of $L$, and a Kähler form $\tilde{\omega}$ on $\mathbf{P}^{N}$ representing $c_{1}\left(\mathbf{P}^{N}\right)$, such that

$$
\omega=\frac{1}{k m} \phi_{L^{m}}^{*}(\tilde{\omega})
$$

If $\tilde{h}$ is a metric on $O(1)$ such that $\tilde{\omega}=\operatorname{Ric}(\tilde{h}) \equiv-\frac{\sqrt{-1}}{2 \pi} \partial \bar{\partial} \tilde{h}$, then we define a metric $h$ on $L$ as follows:

$$
h=\frac{1}{m} \phi_{L^{m}}^{*}(\tilde{h})
$$

A vector field $X$ on $M_{\text {reg }}$ is admissible if there exists an embedding as above, and a holomorphic vector field $\xi$ on $\mathbf{P}^{N}$ such that $\xi$ is tangent to $M_{\text {reg }}$ with $\left.\xi\right|_{M_{\text {reg }}}=X$. The space of all admissible vector fields will be denoted $\eta(M)$. It is the Lie algebra of the algebraic group $G=A u t(M)$ consisting of all invertible algebraic maps from $M$ to itself.

Suppose $\omega$ is an admissible Kähler form on $M_{r e g}$ and $X \in \eta(M)$. Define

$$
F(X)=(n+1) \int_{M_{r e g}} X(f) \omega^{n}
$$


where $f$ is a smooth function on $M_{\text {reg }}$ chosen so that

$$
\operatorname{Ric}(\omega)-\omega=\frac{\sqrt{-1}}{2 \pi} \partial \bar{\partial} f
$$

If $M$ is smooth, then Futaki [F83] has shown that (2.3) is well defined, independent of the choice of $\omega$, and $F: \eta(M) \rightarrow \mathbf{C}$ is a Lie algebra character. For arbitrary Q-Fano $M$, this was proved, using resolution of singularities, by Ding-Tian [DT]. When $M$ is smooth, it is known, through the work of Futaki, Mabuchi and Morita (see [F87], [Ma], [F-Mo]) that $F$ has a lift to a group character $\hat{F}: G \rightarrow \mathbf{C}^{\times}$. They show, using the theory of ChernSimons invariants, that $\hat{F}$ may be constructed as the fiber integral of the Godbillon-Vey class of a certain locally trivial $M$ bundle over the classifying space $B G$. Yotov [Yo99] was able to construct $\hat{F}$ in the general case of QFano varieties by combining the techniques of the previous authors with the Edidin-Graham [EG] theory of equivariant Chow cohomology groups.

Our first theorem gives an alternate construction of $\hat{F}$ in terms of the Deligne pairing (for background on Deligne pairings, see $\S 2.3$ ): Let $M$ be a Q-Fano variety and let $L$ be the extension of $K^{-k}$ from $M_{\text {reg }}$ to $M$. Then the action of $G$ on $M$ lifts canonically to an action on $K^{-1}$ and thus $G$ acts on the pairing $\mathcal{F}=\langle L, L, \ldots, L\rangle$, the Deligne pairing of $L$ with itself $n+1$ times. Since $\mathcal{F}$ is a one dimensional vector space, the action of $G$ on $\mathcal{F}$ defines a group character

$$
\hat{F}: G \rightarrow \operatorname{Aut}(\mathcal{F})=\mathbf{C}^{\times}
$$

Thus, if $\ell_{0}, \ldots, \ell_{n}$ are rational sections of $L$ with $\operatorname{div}\left(\ell_{0}\right) \cap \cdots \cap \operatorname{div}\left(\ell_{n}\right)=\emptyset$, and $\sigma \in G$, then $\hat{F}(\sigma)=\left\langle\sigma_{*} \ell_{0}, \ldots, \sigma_{*} \ell_{n}\right\rangle /\left\langle\ell_{0}, \ldots, \ell_{n}\right\rangle \in \mathbf{C}^{\times}$.

Theorem 1. The character $\hat{F}: G \rightarrow A u t(\mathcal{F})=\mathbf{C}^{\times}$is a lifting of the Futaki character. More precisely, if $X \in h(M)$ and if $X_{\mathbf{R}}=X+\bar{X}$ is the real part of $X$, then

$$
\hat{F}\left(\exp \left(t X_{\mathbf{R}}\right)\right)=\exp \left(t k^{n+1} F(X)\right)
$$

As a corollary, we find that $F(X)$ is the eigenvalue of the Chow point of $M$ : More, precisely, there is a natural action of $G$ on $H^{0}\left(L^{k}\right)$, inducing an action on $\operatorname{Gr}\left(N-n-1, \mathbf{P}^{N}\right)$ and $H^{0}(G r, O(d))$, where $d$ is the degree of $M \subseteq \mathbf{P}^{N}$, and $G r=G r\left(N-n-1, \mathbf{P}^{N}\right)$ is the Grassmannian manifold consisting of all $N-n-1$ planes in $\mathbf{P}^{N}$. Let $\operatorname{Chow}_{k}(M) \in H^{0}(G r, O(d))$ be a representative of the Chow point. Since the Chow point is unique up to scalar multiplication, we have, for each $\sigma \in G$,

$$
\sigma \cdot \operatorname{Chow}_{k}(M) \equiv \operatorname{Chow}_{k}(M) \circ \sigma^{-1}=C(\sigma) \operatorname{Chow}_{k}(M)
$$


for some $C(\sigma) \in \mathbf{C}^{\times}$. The corollary states that $C(\sigma)=\hat{F}(\sigma)^{k^{n+1}}$ :

Corollary 1. The Futaki group character $\hat{F}(\sigma)$ is characterized by

$$
\sigma \cdot \operatorname{Chow}_{k}(M)=\hat{F}(\sigma)^{k^{n+1}} \operatorname{Chow}_{k}(M)
$$

and the Futaki invariant $F(X)$ by

$$
X \cdot \operatorname{Chow}_{k}(M)=k^{n+1} F(X) \operatorname{Chow}_{k}(M)
$$

for all $\sigma \in G \subseteq G L(N+1, \mathbf{C})$, and for all $X \in \operatorname{Lie}(G)$.

\subsection{The Deligne pairing.}

We recall some of the basic definitions and properties in [De] and [Z] ${ }^{1}$ : Let $\pi: \mathcal{X} \rightarrow S$ be a flat projective morphism of integral schemes of relative dimension $n$. Thus for every $s \in S$, the fiber $\mathcal{X}_{s}$ is a projective variety in $\mathbf{P}^{N}$ of dimension $n$. Let $\mathcal{L}_{0}, \mathcal{L}_{1}, \ldots, \mathcal{L}_{n}$ be line bundles on $\mathcal{X}$. The Deligne pairing is a line bundle on $S$, denoted $\left\langle\mathcal{L}_{0}, \mathcal{L}_{1}, \ldots, \mathcal{L}_{n}\right\rangle(\mathcal{X} / S)$, and defined as follows: Let $U \subseteq S$ be a small open set and let $l_{i}$ be a rational section of $\mathcal{L}_{i}$ over $\pi^{-1} U$. Thus $l_{i}: \pi^{-1}(U) \rightarrow \mathcal{L}_{i}$ is a rational function with the property $p_{i} \circ l_{i}=\mathbf{1}_{\pi^{-1} U}$, where $p_{i}: \mathcal{L}_{i} \rightarrow \mathcal{X}$ is the projection map of $\mathcal{L}_{i}$. Assume that the $l_{i}$ are chosen in "general position": This means $\cap_{i} \operatorname{div}\left(l_{i}\right)=\emptyset$ and for each $s$ and $i$ with $s \in U$ and $0 \leq i \leq N$, the fiber $\mathcal{X}_{s}$ is not contained in $\operatorname{div}\left(l_{i}\right)$. Then for every $k$, the map $\left(\cap_{i \neq k} \operatorname{div}\left(l_{i}\right)\right) \rightarrow S$ is finite: For every $s$, $\left(\cap_{i \neq k} \operatorname{div}\left(l_{i}\right)\right) \cap \mathcal{X}_{s} \subseteq \mathcal{X}_{s}$ is a zero cycle $\sum n(s) P(s)$. This means that the $n(s)$ are integers and the points $P(s)$ are a finite set of points in $\mathcal{X}_{s}$.

Now we define $\left\langle\mathcal{L}_{0}, \mathcal{L}_{1}, \ldots, \mathcal{L}_{n}\right\rangle(\mathcal{X} / S)$. Over a small $U \subseteq S$, this line bundle is trivial and generated by the symbol $\left\langle l_{0}, \ldots, l_{n}\right\rangle$ where the $l_{i}$ are chosen to be in general position. If $l_{i}^{\prime}$ is another set of rational sections in general position, then $\left\langle l_{0}^{\prime}, \ldots, l_{n}^{\prime}\right\rangle=\psi(s)\left\langle l_{0}, \ldots, l_{n}\right\rangle$ for some nowhere vanishing function $\psi$ on $U$ which we must specify. We do this one section at a time: Assume that $l_{i}=l_{i}^{\prime}$ for all $i \neq k$. Assume as well that the rational function $f_{k}=l_{k}^{\prime} / l_{k}$ is well defined and non-zero on $\left(\cap_{i \neq k} \operatorname{div}\left(l_{i}\right)\right)$. Then $\psi(s)=$ $\prod f(P(s))^{n(s)}$. The Deligne pairing is obviously multilinear in the bundles $\mathcal{L}_{0}, \cdots, \mathcal{L}_{n}$. Applied to a family $\mathcal{X}$ consisting of a single irreducible normal variety (i.e. $S$ consists of a single point), it produces a one-dimensional vector space.

\footnotetext{
${ }^{1}$ At the December 2002 Complex Geometry conference in Tokyo, Professors T. Mabuchi and L. Weng informed us that they have also been aware for some time of potential applications of Deligne pairings to Kähler geometry.
} 
Let $\pi: \mathcal{X} \rightarrow S$ and $\mathcal{L}_{0}, \ldots, \mathcal{L}_{n}$ as above. Let $l$ be a rational section of $\mathcal{L}_{n}$. Assume all components of $\operatorname{div}(l)$ are flat over $S$. Then we have the following induction formula:

$$
\left\langle\mathcal{L}_{0}, \ldots, \mathcal{L}_{n}\right\rangle(\mathcal{X} / S)=\left\langle\mathcal{L}_{0}, \ldots, \mathcal{L}_{n-1}\right\rangle(\operatorname{div}(l) / S)
$$

where $\left\langle\mathcal{L}_{0}, \ldots, \mathcal{L}_{n-1}\right\rangle(Z / S)=\prod_{i}\left\langle\mathcal{L}_{0}, \ldots, \mathcal{L}_{n-1}\right\rangle\left(Y_{i} / S\right)^{n_{i}}$ if $Z=\sum_{i} n_{i} Y_{i}$ is a cycle on $S$.

Assume now that $\mathcal{X}, S$ are defined over $\mathbf{C}$, and that $\mathcal{L}_{i}$ is endowed with a smooth hermitian metric (that is, for any holomorphic map from a smooth variety $Y$ to $\mathcal{X}$, the pull back metric on $f^{*} \mathcal{L}$ is smooth on $\left.Y\right)$. We now define a hermitian metric on $\left\langle\mathcal{L}_{0}, \ldots, \mathcal{L}_{n}\right\rangle(\mathcal{X} / S)$ : Let

$$
c_{1}^{\prime}\left(\mathcal{L}_{i}\right)=-\frac{\sqrt{-1}}{2 \pi} \partial \bar{\partial} \log \|l\|^{2}
$$

be the normalized curvature of $\mathcal{L}_{i}$ where $l$ is a local invertible section of $\mathcal{L}_{i}$. Then $c_{1}^{\prime}\left(\mathcal{L}_{i}\right)$ is a $(1,1)$ form on $\mathcal{X}_{\text {reg }}$. The metric on $\left\langle\mathcal{L}_{0}, \ldots, \mathcal{L}_{n}\right\rangle(\mathcal{X} / S)$ is defined by induction: When $n=0,\langle\mathcal{L}\rangle_{s}=\otimes_{p \in \pi^{-1}(s)} \mathcal{L}_{p}$ so we define

$$
\left\|\left\langle l_{0}\right\rangle\right\|_{s}=\prod_{p \in \pi^{-1}(s)}\left\|l_{0}(p)\right\|
$$

In general, we define

$\log \left\|\left\langle l_{0}, \ldots, l_{n}\right\rangle\right\|=\log \left\|\left\langle l_{0}, \ldots, l_{n-1}\right\rangle\left(\operatorname{div}\left(l_{n}\right) / S\right)\right\|+\int_{\mathcal{X} / S} \log \left\|l_{n}\right\| \Lambda_{i=0}^{n-1} c_{1}^{\prime}\left(\mathcal{L}_{i}\right)$

where the integral is the fiber integral over $S$, so both sides are functions on the base manifold $S$.

If we combine the induction formula with the definition of the metric, we immediately get the following isometry:

$$
\begin{gathered}
\left\langle\mathcal{L}_{0}, \ldots, \mathcal{L}_{n}\right\rangle(\mathcal{X} / S)= \\
\left\langle\mathcal{L}_{0}, \ldots, \mathcal{L}_{n-1}\right\rangle(\operatorname{div}(l) / S) \otimes \mathcal{O}\left(-\int_{\mathcal{X} / S} \log \left\|l_{n}\right\| \Lambda_{i=0}^{n-1} c_{1}^{\prime}\left(\mathcal{L}_{i}\right)\right)
\end{gathered}
$$

where $\mathcal{O}(f)$ denotes the trivial line bundle with metric $\|1\|=\exp (-f)$. In particular,

$$
\left\langle\mathcal{L}_{0}, \ldots, \mathcal{L}_{n-1}, \mathcal{L}_{n} \otimes \mathcal{O}(\phi)\right\rangle(\mathcal{X} / S)=\left\langle\mathcal{L}_{0}, \ldots, \mathcal{L}_{n}\right\rangle(\mathcal{X} / S) \otimes \mathcal{O}(E)
$$


where

$$
E=\int_{X / S} \phi \cdot \prod_{k<n} c_{1}^{\prime}\left(\mathcal{L}_{k}\right)
$$

Using induction we get the following change of metric formula:

$$
\left\langle\mathcal{L}_{0} \otimes \mathcal{O}\left(\phi_{0}\right), \ldots, \mathcal{L}_{n} \otimes \mathcal{O}\left(\phi_{n}\right)\right\rangle(\mathcal{X} / S)=\left\langle\mathcal{L}_{0}, \ldots, \mathcal{L}_{n}\right\rangle(\mathcal{X} / S) \otimes \mathcal{O}(E)
$$

where

$$
E=\int_{\mathcal{X} / S} \sum_{j=0}^{n} \phi_{j} \cdot \prod_{k<j} c_{1}^{\prime}\left(\mathcal{L}_{k} \otimes \mathcal{O}\left(\phi_{k}\right)\right) \cdot \prod_{k>j} c_{1}^{\prime}\left(\mathcal{L}_{k}\right)
$$

Let $\phi: \mathcal{X} \rightarrow \mathcal{Y}$ be a morphism of projective flat integral schemes over $S$ and let $m=\operatorname{dim}(\mathcal{X} / \mathcal{Y})$ and $n=\operatorname{dim}(\mathcal{Y} / S)$. If $\mathcal{K}_{0}, \ldots, \mathcal{K}_{m}$ are line bundles on $\mathcal{X}$ and $\mathcal{L}_{1}, . ., \mathcal{L}_{n}$ are line bundles on $\mathcal{Y}$ then we have a canonical isometry:

$$
\begin{aligned}
\left\langle\mathcal{K}_{0}, \ldots,\right. & \left.\mathcal{K}_{m}, \phi^{*} \mathcal{L}_{1}, \ldots, \phi^{*} \mathcal{L}_{n}\right\rangle(\mathcal{X} / S)= \\
& \left\langle\left\langle\mathcal{K}_{0}, \ldots, \mathcal{K}_{m}\right\rangle(\mathcal{X} / \mathcal{Y}), \mathcal{L}_{1}, \ldots, \mathcal{L}_{n}\right\rangle(\mathcal{Y} / S)
\end{aligned}
$$

Now if $\mathcal{N}$ and $\mathcal{N}^{\prime}$ are hermitian line bundles on $S$, then it follows easily from the definition of the Deligne pairing that we have the following isometries

$$
\left\langle\mathcal{L}_{1}, \ldots, \mathcal{L}_{n}, \pi^{*} \mathcal{N}\right\rangle(\mathcal{Y} / S)=\mathcal{N}^{\operatorname{deg}\left[c_{1}\left(\mathcal{L}_{1 \eta}\right) \cdots c_{1}\left(\mathcal{L}_{n \eta}\right)\right]}
$$

and

$$
\left\langle\mathcal{L}_{1}, \ldots, \mathcal{L}_{n-1}, \pi^{*} \mathcal{N}, \pi^{*} \mathcal{N}^{\prime}\right\rangle(\mathcal{Y} / S)=\mathcal{O}_{S}
$$

Here $L_{j \eta}$ is the restriction of $\mathcal{L}_{j}$ to a generic point of $S$. In particular, if $\mathcal{K}_{0}=$ $\phi^{*} \mathcal{L}_{0}$ in $(2.11)$, then the right side reduces to $\left\langle\mathcal{L}_{0}, \ldots, \mathcal{L}_{n}\right\rangle{ }^{\operatorname{deg}\left[c_{1}\left(\mathcal{K}_{1 \eta}\right) \cdots c_{1}\left(\mathcal{K}_{m \eta}\right)\right]}$, where $\mathcal{K}_{j \eta}$ is the restriction of $\mathcal{K}_{j}$ to the fiber at a generic point of $\mathcal{Y}$.

\subsection{Energy Functionals and the Deligne Pairing.}

The change of metric formula (2.10) can be used to show that various important functionals have a natural interpretation as metrics on certain Deligne pairings. This point of view is rather useful (cf. [PS1] and [PS2] ) and will be adopted as well in the proofs of the theorems in this paper. In this section we recall some of the key functionals and give their metric interpretation: Let $M$ be a Kähler manifold with Kähler form $\omega$. Denote by

$$
P(M, \omega)=\left\{\phi \in C^{\infty}(M, \mathbf{R}): \omega_{\phi}=\omega+\frac{\sqrt{-1}}{2 \pi} \partial \bar{\partial} \phi>0\right\}
$$


the space of Kähler potentials, and recall that $\operatorname{Ric}(\omega)=-\frac{\sqrt{-1}}{2 \pi} \partial \bar{\partial} \omega^{n}$ is the Ricci curvature form of $\omega$. The Aubin-Yau functional is defined by

$$
\mathrm{AY}_{\omega}(\phi)=\frac{1}{\left[\omega^{n}\right]} \int_{M} \sum_{k=0}^{n} \phi \omega^{n-k} \omega_{\phi}^{k}
$$

the Futaki functional is defined by

$$
\operatorname{Fut}_{\omega}(\phi)=\frac{1}{\left[\omega^{n}\right]} \int_{M} \sum_{k=0}^{n} \phi \operatorname{Ric}(\omega)^{n-k} \operatorname{Ric}\left(\omega_{\phi}\right)^{k}
$$

and the Mabuchi K-energy is defined by

$$
\begin{aligned}
\nu_{\omega}(\phi)=\frac{1}{\left[\omega^{n}\right]}[ & \int_{M}\left(\log \frac{\omega_{\phi}^{n}}{\omega^{n}}\right) \cdot \omega_{\phi}^{n}-\sum_{i=0}^{n-1} \int_{M} \phi \operatorname{Ric}(\omega) \omega_{\phi}^{i} \omega^{n-1-i} \\
& \left.+\frac{n c_{1}(M)[\omega]^{n-1}}{(n+1)[\omega]^{n}} \cdot \sum_{i=0}^{n} \int_{M} \phi \omega_{\phi}^{i} \omega^{n-i}\right]
\end{aligned}
$$

(With a different normalization, the Aubin-Yau functional appears in the literature as the functional $F_{\omega}^{0}(\phi)=-\frac{1}{n+1} \mathrm{AY}_{\omega}(\phi)$.) Now suppose that $L \rightarrow$ $M$ is a holomorphic line bundle with metric $h$ and $\omega=\operatorname{Ric}(h)=-\frac{\sqrt{-1}}{2 \pi} \partial \bar{\partial} h$ (so in particular, $\omega \in c_{1}(L)$ ). Corresponding to the three functions (2.14), (2.15) and (2.16), we define the metrized $\mathbf{Q}$ line bundles:

$$
\begin{gathered}
\mathcal{A}_{h}=\langle L, \ldots, L\rangle^{\frac{1}{c_{1}(L)^{n}}}, \quad \mathcal{F}_{h}=\left\langle K^{-1}, K^{-1}, \ldots, K^{-1}\right\rangle \\
\mathcal{M}_{h}=\langle K, L, \ldots, L\rangle^{\frac{1}{c_{1}(L)^{n}}}\langle L, \ldots, L\rangle^{\frac{n}{n+1} \frac{c_{1}(M) c_{1}(L)^{n-1}}{\left[c_{1}(L)^{n}\right]^{2}}}
\end{gathered}
$$

Then a direct calculation from the change of metric formula (2.10) gives

Theorem 2. The Aubin-Yau, Futaki, and Mabuchi energy functionals arise as changes of metrics in the corresponding Deligne pairings

$$
\begin{gathered}
\mathcal{A}_{h e^{-\phi}}=\mathcal{A}_{h} \otimes O\left(\mathrm{AY}_{\omega}(\phi)\right), \quad \mathcal{F}_{h e^{-\phi}}=\mathcal{F}_{h} \otimes O\left(\operatorname{Fut}_{\omega}(\phi)\right) \\
\mathcal{M}_{h e^{-\phi}}=\mathcal{M}_{h} \otimes O\left(\nu_{\omega}(\phi)\right)
\end{gathered}
$$




\subsection{Proof of Theorem 1 .}

For notational simplicity, we give the proof in the case $k=1$.

Fix $h$, a smooth metric on $L=K^{-1}$. If $\phi$ is a smooth function on $M$, then $L(\phi)$ is the bundle $L$ equipped with the metric $h e^{-\phi}$. If $\sigma \in G$, then $\sigma: L \rightarrow L\left(\phi_{\sigma}\right)$ is an isometry for a uniquely defined smooth function $\phi_{\sigma}$. Here "smooth" means that for every holomorphic map $f$ from a complex manifold $Y$ to $M$, the pull back metric $f^{*} h$ is a smooth metric on $f^{*} L$ and the pull back function $\phi_{\sigma} \circ f$ is a smooth function on $Y$. For example, one can construct such an $h$ via formula (2.2).

Now $F\left(\sigma_{t}\right):\langle L, \ldots, L\rangle \rightarrow\left\langle L\left(\phi_{t}\right), \ldots, L\left(\phi_{t}\right)\right\rangle=\langle L, \ldots, L\rangle(E(t))$ is an isometry, where $\sigma_{t}=\exp \left(t X_{\mathbf{R}}\right)$. Thus by $(2.10)$

$$
E(t)=\sum_{j=0}^{n} \int_{M_{r e g}} \phi_{t} \omega_{t}^{j} \omega^{n-j}
$$

Here $\omega_{t}=\omega+\frac{\sqrt{-1}}{2 \pi} \partial \bar{\partial} \phi_{t}$ and $\phi_{t}=\phi_{\sigma_{t}}$.

Now let $s \in\langle L, \ldots, L\rangle$. Then $F\left(\sigma_{t}\right)(s)=\hat{F}\left(\sigma_{t}\right) \cdot s$ so $\left|\hat{F}\left(\sigma_{t}\right)\right| \cdot|s|=$ $\left|F\left(\sigma_{t}\right)(s)\right|=|s| \exp (E(t))$. Since $F\left(\sigma_{t}\right)$ is a homomorphism $R \rightarrow \mathbf{C}^{\times}$, we have $F\left(\sigma_{t}\right)=\exp (t G(X))$ with $G(X) \in \mathbf{C}$, so

$$
E(t)=\log \left|F\left(\sigma_{t}\right)\right|=t \operatorname{Re}(G(X))
$$

In other words, $\operatorname{Re}(G(X))=E^{\prime}(t)$. On the other hand, differentiating both sides of (2.19):

$$
E^{\prime}(t)=\sum_{j=0}^{n} \int_{M_{r e g}} \dot{\phi}_{t} \omega_{t}^{j} \omega^{n-j}+\sum_{j=0}^{n} \int_{M_{r e g}} \phi_{t} \frac{\sqrt{-1}}{2 \pi} \partial \bar{\partial} \dot{\phi}_{t} j \omega_{t}^{j-1} \omega^{n-j}
$$

Integrating by parts in the second integral, we obtain

$$
E^{\prime}(t)=\int_{M_{r e g}} \dot{\phi}_{t} \omega^{n}=\operatorname{Re}(F(X))
$$

The last equality is proved using integration by parts (see p. 23 of [FMS] for the proof for smooth manifolds, and [Yo99] for the general case). We conclude that $\operatorname{Re}(F(X))=\operatorname{Re}(G(X))$. Thus $-\operatorname{Im}(F(X))=\operatorname{Re}(i F(X))=$ $\operatorname{Re}(F(i X))=\operatorname{Re}(G(i X))$. To prove Theorem 1, we must show that $G(i X)=$ $i G(X)$, that is, we must show that $F: G \rightarrow \mathbf{C}^{\times}$is a holomorphic function. This follows from the following: 
Lemma. The function $F: G \rightarrow \mathbf{C}^{\times}$is an algebraic map, that is, $F$ is locally defined by rational functions.

Proof. We may assume that $G$ is an infinite group. Let $\left\langle\ell_{0}, \ldots, \ell_{n}\right\rangle$ be a section of $\langle L, \ldots, L\rangle$. Thus $\cap_{j=0}^{n} \operatorname{div}\left(\ell_{j}\right)=\emptyset$. Let $\sigma \in G$ be in a small neighborhood of $\mathbf{1}_{\mathbf{G}}$, the identity element of $G$. Define

$$
Z_{j}(\sigma)=\prod_{p<j} \operatorname{div}\left(\sigma \ell_{p}\right) \prod_{p>j} \operatorname{div}\left(\ell_{p}\right)=\sum_{k} n_{j k}(\sigma) y_{j k}(\sigma)
$$

with $n_{j k}(\sigma) \in \mathbf{Z}$ and $y_{j k}(\sigma)$ points in $M$. Then

$$
F(\sigma)^{-1}=\frac{\left\langle\sigma \ell_{0}, \ldots, \sigma \ell_{n}\right\rangle}{\left\langle\ell_{0}, \ldots, \ell_{n}\right\rangle}=\prod_{j=0}^{n} f_{j}(\sigma)
$$

where

$$
f_{j}(\sigma)=\prod_{k}\left[\frac{\sigma \ell_{j}}{\ell_{j}}\right]\left(y_{j k}(\sigma)\right)^{n_{j k}(\sigma)}
$$

We claim that each $f_{j}(\sigma)$ is rational and defined in a neighborhood of $\sigma=$ $\mathbf{1}_{\mathbf{G}}$. To see this, let $\pi: G \times M \rightarrow M$ be the projection map, and let $\mu: G \times M \rightarrow M$ be the map $(\sigma, m) \rightarrow \sigma(m)$. Let $Z_{j} \subseteq G \times M$ be defined by $Z_{j}=\prod_{p<j} \operatorname{div}\left(\mu^{*} \ell_{p}\right) \prod_{p>j} \operatorname{div}\left(\pi^{*} \ell_{p}\right)$. Then $\pi$ restricted to $Z_{j}$ induces a map $\pi_{j}: Z_{j} \rightarrow G$. It has the property $\pi_{j}^{-1}(\sigma)=Z_{j}(\sigma)$. The map $\pi_{j}$ is generically finite and $f_{j}(\sigma)=\operatorname{Norm}_{Z_{j} / G}\left[\frac{\sigma \ell_{j}}{\ell_{j}}\right]$ is therefore rational, which implies that $F(\sigma)$ is rational. Since $F$ is a homomorphism, we conclude that $F$ is everywhere defined, and is therefore regular at every point $\sigma \in G$. This proves the lemma.

Finally, the corollary follows from the theorem of Zhang [Z], which states that $\left\langle L^{k}, \ldots, L^{k}\right\rangle=\langle L, \ldots, L\rangle^{k^{n+1}}$ is canonically isomorphic to the Chow line bundle.

\section{The Futaki invariant of a complete intersection.}

The Futaki invariant of a complete intersection has been computed by $\mathrm{Lu}$ [Lu99] (see as well the paper of Yotov [Yo99]) and is given as follows:

Theorem 3. (Lu) Let $M$ be the $N-s$ dimensional normal Fano variety in $\mathbf{P}^{N}$ defined by $F_{1}=\cdots=F_{s}=0$, where $F_{1}, \cdots, F_{s}$ are homogeneous polynomials of degrees $d_{1}, \ldots, d_{s}$. Let $X=\sum_{i, j=0}^{N} a_{i j} z_{i} \frac{\partial}{\partial z^{j}}$ be a holomorphic vector 
field on $\mathbf{P}^{N}$, normalized so that the matrix $\left(a_{i j}\right)$ has trace zero. Assume

$$
X F_{i}=\kappa_{i} F_{i}, \quad i=1, \ldots, s
$$

for complex constants $\kappa_{1}, \ldots, \kappa_{s}$. Then

$$
F(X)=(N-s+1)\left[m^{N-s} \prod_{i=1}^{s} d_{i}\right] \cdot\left(-\sum_{i=1}^{s} \kappa_{i}+\frac{m}{N-s+1} \sum_{i=1}^{s} \frac{\kappa_{i}}{d_{i}}\right)
$$

where $m=N+1-d_{1}-\cdots-d_{s}$.

We wish to show how Theorem 1 can be used to give a proof of Theorem 3. To do this, it is useful to view $M$ as a fiber in the family of all complete intersections:

Let $R_{d}$ be the space of homogeneous polynomials of degree $d$, and let

$$
Z=\mathbf{P}\left(R_{d_{1}}\right) \times \cdots \times \mathbf{P}\left(R_{d_{s}}\right)
$$

where $s=N-n$. Let

$$
\mathcal{X}=\left\{(z, x): z=\left(F_{1}, \ldots, F_{s}\right) \in Z, x \in \mathbf{P}^{N}, F_{1}(x)=\cdots=F_{s}(x)=0\right\}
$$

Then $\mathcal{X} \subseteq Z \times \mathbf{P}^{N}$ is a smooth variety and $\operatorname{dim}(\mathcal{X})=\operatorname{dim}(Z)+n$. Let $\mathcal{L} \rightarrow \mathcal{X}$ be the pull back of the hyperplane bundle on $\mathbf{P}^{N}$ equipped with the Fubini-Study metric, and let $\mathcal{K} \rightarrow \mathcal{X}$ be the relative canonical bundle of $\mathcal{X} / Z$. Let

$$
Z_{0}=\left\{z \in Z: \pi^{-1} z \quad \text { has dimension } n\right\}
$$

where $\pi: \mathcal{X} \rightarrow Z$ is the natural projection. Then $Z_{0} \subseteq Z$ is Zariski open, and the restricted map $\pi: \mathcal{X}_{0} \rightarrow Z_{0}$ is flat (where $\mathcal{X}_{0}=\pi^{-1}\left(Z_{0}\right)$ ). We want to compute the Deligne pairing

$$
\mathcal{F}=\left\langle\mathcal{K}^{-1}, \mathcal{K}^{-1}, \ldots, \mathcal{K}^{-1}\right\rangle\left(\mathcal{X}_{0} / Z_{0}\right)
$$

which is a line bundle on $Z_{0}$.

Note that $F_{j}(z)$, when viewed as a function of $\left(F_{j}, z\right)$, is homogeneous of degree one in $F_{j}$ and homogeneous of degree $d_{j}$ in $z$. Thus it represents a global section $\sigma_{j}$ of $H_{j} \otimes \mathcal{L}^{d_{j}} \equiv H_{j} \mathcal{L}^{d_{j}}$. The simultaneous vanishing of the $\sigma_{j}$ defines the subvariety $\mathcal{X}_{0} \subseteq Z_{0}$. Thus we have, applying (2.4) successively $s$ times,

$$
\left\langle\mathcal{K}^{-1}, \ldots, \mathcal{K}^{-1}\right\rangle\left(\mathcal{X}_{0} / Z_{0}\right)=\left\langle H_{1} \mathcal{L}^{d_{1}}, \ldots, H_{s} \mathcal{L}^{d_{s}}, \mathcal{K}^{-1}, \ldots \mathcal{K}^{-1}\right\rangle\left(\left(Z_{0} \times \mathbf{P}^{N}\right) / Z_{0}\right)
$$


where $H_{k}$ is the pullback of the hyperplane bundle on $\mathbf{P}\left(R_{d_{k}}\right)$. Next we recall that the relative canonical bundle of $Z_{0} \times \mathbf{P}^{N} \rightarrow Z_{0}$ is $\mathcal{L}^{N+1}$. Since $\mathcal{X}$ is a complete intersection, defined by the sections $\sigma_{1}=\cdots=\sigma_{d}=0$, and since $\sigma_{j}$ is a global section of $\mathcal{L}^{d_{j}} H_{j}$, applying the adjunction formula $s$ times yields

$$
\mathcal{K}^{-1}=\mathcal{L}^{m} H_{1}^{-1} \cdots H_{s}^{-1}
$$

where $m=N+1-d_{1}-\cdots-d_{s}$. Expanding (3.1) using the multi-linearity

of the Deligne pairing, we get the main term, $\langle\mathcal{L}, \ldots \mathcal{L}\rangle^{d_{1} \cdots d_{s} \cdot m^{n+1}}$, which is the trivial line bundle: Indeed, $\left\langle z_{0}, z_{1}, \ldots, z_{N}\right\rangle$ is a global nowhere vanishing section of $\langle\mathcal{L}, \ldots, \mathcal{L}\rangle$. As for the other terms in the expansion of (3.1): If two or more of the $H_{i}$ appear, say $H_{i}$ and $H_{j}$, we get the trivial bundle, by applying (2.13), with $\mathcal{N}=H_{i}$ and $\mathcal{N}^{\prime}=H_{j}$. Thus the only terms which contribute are those with one $H$. There are two ways this can happen: Either the $H_{i}$ comes from the $i^{\text {th }}$ position in (3.1), with $1 \leq i \leq s$, and then it is paired with $\mathcal{L}^{d_{k}}$ with $k \neq i$, as well as $n+1=N-s+1$ copies of $\mathcal{L}^{m}$. Or $H_{i}$ comes from any one of the last $n+1$ positions and then it is paired with $\mathcal{L}^{d_{r}}, 1 \leq r \leq s$ as well as $n=N-s$ copies of $\mathcal{L}^{m}$. In the first case we apply (2.12) with $\mathcal{N}=H_{i}$ and $\left\{\mathcal{L}_{1}, \ldots, \mathcal{L}_{n}\right\}=\left\{\mathcal{L}^{d_{1}}, \ldots, \mathcal{L}^{d_{i-1}}, \mathcal{L}^{d_{i+1}}, \ldots, \mathcal{L}^{d_{s}}, \mathcal{L}^{m}, \ldots, \mathcal{L}^{m}\right\}$ with $\mathcal{L}^{m}$ repeated $N-s+1$ times. In the second case we apply (2.12) $N-s+1=n+1$ times with $\mathcal{N}=H_{i}^{-1}$ and $\left\{\mathcal{L}_{1}, \ldots, \mathcal{L}_{n}\right\}=\left\{\mathcal{L}^{d_{1}}, \ldots, \mathcal{L}^{d_{s}}, \mathcal{L}^{m}, \ldots, \mathcal{L}^{m}\right\}$ where $\mathcal{L}^{m}$ is repeated $N-s$ times. Thus we obtain

$$
\left\langle H_{1} \mathcal{L}^{d_{1}}, \ldots, H_{s} \mathcal{L}^{d_{s}}, \mathcal{L}^{m} H_{1}^{-1} \cdots H_{s}^{-1}, \ldots, \mathcal{L}^{m} H_{1}^{-1} \cdots H_{s}^{-1}\right\rangle=\prod_{i=1}^{s} H_{i}^{a_{i}}
$$

where

$$
a_{i}=(-1)(n+1) m^{N-s} d_{1} d_{2} \cdots d_{s}+m^{N-s+1}\left(d_{1} d_{2} \cdots d_{s}\right) / d_{i}
$$

Combining (3.1) and (3.2) we obtain $F(X)=\sum a_{i} \kappa_{i}$ and this, together with formula (3.3) yields Theorem 3.

\section{Energy functionals for a complete intersection.}

\subsection{The adjunction formula with metrics.}

We wish to prove a non-linear version of Theorem 3 in which the Mabuchi $\mathrm{K}$-energy is expressed in terms of certain norms on the polynomials defining $M$. To do this, we first prove a metrized version of the adjunction formula: 
Let $X$ be a smooth variety and $Y \subseteq X$ a smooth subvariety. The adjunction formula says

$$
K_{Y}=\left.\left(K_{X} \otimes O(Y)\right)\right|_{Y}
$$

where $O(Y)$ is the line bundle associated to the divisor $Y$.

The isomorphism is easy to describe in local coordinates: Let $y \in Y$. Choose local coordinates $\left(z_{1}, \ldots, z_{n}\right)$ in such a way that $y$ is the origin, and $Y$ is the set $z_{1}=0$. Then a local section of $K_{X} \otimes O(Y)$ is meromorphic differential form of the type

$$
\eta=\frac{g(z) d z_{1} \wedge d z_{2} \wedge \cdots \wedge d z_{n}}{z_{1}}
$$

where $g(z)=g\left(z_{1}, \ldots, z_{n}\right)$ is a holomorphic function. The isomorphism (4.1) is $\eta \mapsto \theta$ where

$$
\theta=g\left(0, z_{2}, \ldots, z_{n}\right) d z_{2} \wedge \cdots d z_{n}
$$

that is, $\theta$ is the residue of $\eta$. Note that the map $\eta \mapsto \theta$ does not depend on the choice of coordinates: If $z_{1}=u z_{1}^{\prime}$ where $u$ is a nowhere vanishing function, then $\frac{d z_{1}}{z_{1}}=\frac{d z_{1}^{\prime}}{z_{1}^{\prime}}+d(\log u)$ and thus $\eta=\frac{g\left(z^{\prime}\right) d z_{1}^{\prime} \wedge d z_{2} \wedge \cdots \wedge d z_{n}}{z_{1}^{\prime}}+$ $\frac{g\left(z^{\prime}\right) z_{1}^{\prime} d(\log u) \wedge d z_{2} \wedge \cdots \wedge d z_{n}}{z_{1}^{\prime}}$. In other words,

$$
\eta=\frac{\left[g\left(z^{\prime}\right)+z_{1}^{\prime} \frac{\partial \log u}{\partial z_{1}^{\prime}}\right] d z_{1}^{\prime} \wedge d z_{2} \wedge \cdots \wedge d z_{n}}{z_{1}^{\prime}}
$$

where $z^{\prime}=\left(z_{1}^{\prime}, z_{2}, \ldots, z_{n}\right)$. But $z_{1}^{\prime} \frac{\partial \log u}{\partial z_{1}^{\prime}}$ vanishes on $Y$. This shows the isomorphism (4.1) is well defined.

An equivalent way of formulating (4.1) is

$$
\left.K_{Y} \otimes O(-Y)\right|_{Y}=\left.\left(K_{X}\right)\right|_{Y}
$$

Again, this is easy to describe in local coordinates: Let $y \in Y$, let $U \subseteq X$ be an open set containing $y$, and let $W=U \cap Y \subseteq Y$. As before, we assume that $Y$ is locally given by the equation $z_{1}=0$. Then a section of $K_{X}$ over $U$ is a differential form $\eta=g(z) d z_{1} \wedge \cdots \wedge d z_{n}$ with $g$ holomorphic on $U$. We say $\eta$ is equivalent to zero if $g\left(0, z_{2}, \ldots, z_{n}\right)=0$. If $\eta$ and $\eta^{\prime}$ are sections of $K_{X}$ over $U$ and $U^{\prime}$ respectively, where $U \cap Y=U^{\prime} \cap Y=W$, we say that $\eta \sim \eta^{\prime}$ if $\eta-\eta^{\prime}$ is equivalent to zero. A section of $\left.\left(K_{X}\right)\right|_{Y}$ over $W$ is then an equivalence class $[\eta]$. Similarly, a section of $O(-Y)$ is a holomorphic function 
$f$ on $U$ such that $f\left(0, z_{2}, \ldots, z_{n}\right)=0$, in other words, $f$ is divisible by $z_{1}$. We say that $f$ is equivalent to zero if it is divisible by $z_{1}^{2}$. A section of $\left.O(-Y)\right|_{Y}$ is then an equivalence class $[f]$. Finally a section of $\left.K_{Y} \otimes O(-Y)\right|_{Y}$ is locally given by an expression of the form $[f] d z_{2} \wedge \cdots \wedge d z_{n}$. And now (4.2) is given by $[\eta] \mapsto[f] d z_{2} \wedge \cdots \wedge d z_{n}$, where $f=z_{1} g$.

Now we want prove the adjunction formula with metrics: Let $\omega$ be a Kähler metric on $X$. Then $\omega^{n}$ is a metric on $K_{X}^{-1}$, and $\omega^{n-1}$ is a metric on $K_{Y}$. We want to construct a metric $\|\cdot\|_{a d}$ on $\left.O(Y)\right|_{Y}$ in such a way that (4.1) becomes an isometry. In other words, for every $y \in Y$, we want $\|\cdot\|_{a d}$ to have the property

$$
\left\|\frac{1}{z_{1}}\right\|_{a d}=1
$$

at the origin, if $\left(z_{1}, \ldots, z_{n}\right)$ are local coordinates on $U \subseteq X$, centered at $y \in Y$, satisfying

a) $Y \cap U=\left\{z: z_{1}=0\right\}$.

b) $\omega=\frac{\sqrt{-1}}{2 \pi} \sum_{j} d z_{j} \wedge d \bar{z}_{j}$ at the origin.

This is equivalent to constructing a metric $\|\cdot\|_{a d}$ on $\left.O(-Y)\right|_{Y}$ such that $\left\|z_{1}\right\|_{a d}=1$ at the origin, if $\left(z_{1}, \ldots, z_{n}\right)$ satisfy a) and b). We do this as follows:

Let $t$ be a section of $O(-Y)$ over some open set $W \subseteq Y$. Thus $t$ is represented by a holomorphic function $T$ on $U \subseteq X$, with $U \cap X=W$, with the property: $T$ vanishes on $Y$. Here two holomorphic functions on $U$ are "equivalent" if their difference vanishes to order at least two on $Y$. Define

$$
\|t\|_{a d}^{2}=\left.\left(\frac{\omega^{n-1} \wedge \frac{\sqrt{-1}}{2 \pi} \partial \bar{\partial}\left(|T|^{2}\right)}{\omega^{n}}\right)\right|_{Y}=\left\|\left.\left(\nabla_{\omega} T\right)\right|_{Y}\right\|^{2}=\left\|\left.(\partial T)\right|_{Y}\right\|^{2}
$$

where $\nabla_{\omega} T$ is the gradient of $T$ with respect to the metric $\omega$. This makes sense since $|T|^{2}$ is a smooth function on $X$. Thus $\|T\|_{a d}^{2}$ is a smooth function on $Y$.

The following theorem is closely related to Proposition 3.1 of [Lu99], although the statement there looks rather different:

Theorem 4. Let $(X, \omega)$ be a Kähler manifold and $Y \subseteq X$ a smooth submanifold of codimension one. Let $t$ be a section of $\left.O(-Y)\right|_{Y}$ over some open set $W \subseteq Y$. Thus $t$ is an equivalence class represented by a holomorphic function $T$ on $U \subseteq X$, with $U \cap X=W$, and $\left.T\right|_{Y}=0$ (here two holomorphic 
functions on $U$ are "equivalent" if their difference vanishes to order at least two on $Y)$. Define

$$
\|t\|_{a d}^{2}=\left.\left(\frac{\omega^{n-1} \wedge \frac{\sqrt{-1}}{2 \pi} \partial \bar{\partial}\left(|T|^{2}\right)}{\omega^{n}}\right)\right|_{Y}=\left\|\left.\left(\nabla_{\omega} T\right)\right|_{Y}\right\|^{2}=\left\|\left.(\partial T)\right|_{Y}\right\|^{2}
$$

where $\nabla_{\omega} T$ is the gradient of $T$ with respect to the Kähler form $\omega$. Then $\|\cdot\|_{a d}$ is a well defined metric on $\left.O(-Y)\right|_{Y}$ and

$$
\left.K_{Y} \otimes O(-Y)\right|_{Y}=\left.\left(K_{X}\right)\right|_{Y}
$$

is an isometry with respect to this metric.

Note that (4.4) makes sense since $|T|^{2}$ is a smooth function on $X$. Thus $\|T\|_{a d}^{2}$ is a smooth function on $Y$.

Proof. First observe that the norm $\|\cdot\|_{a d}$ has a very simple description in local coordinates: Let $y \in Y$ and let $\left(z_{1}, \ldots, z_{n}\right)$ be local coordinates centered at $y$ satisfying a) and b). We write $T=z_{1} F$ where $F$ is holomorphic on $U$. Then

$$
\left(\left.|| t\right|_{a d} ^{2}\right)(y)=\left(|F|^{2}\right)(y)
$$

Note that (4.6) holds only at the point $y$, whereas (4.4) holds on all of $W$.

To establish Theorem 4 we must prove the following:

1) $\|t\|_{\text {ad }}$ depends only on the equivalence class of $t$.

2) If $f$ is a holomorphic function on $W \subseteq Y$, then $\|f t\|_{a d}=|f| \cdot\|t\|_{a d}$.

3) $\|t\|_{a d} \geq 0$ with equality if and only if $t=0$.

4) If $\left(z_{1}, \ldots, z_{n}\right)$ are coordinates centered at a point $y \in Y$ satisfying a) and $\mathrm{b}$ ), and if $Y$ is defined by $z_{1}=0$, then $\left\|z_{1}\right\|_{a d}=1$ (or, more precisely, $\|t\|_{a d}=1$ where $t$ is the section of $O(-Y)$ represented by $\left.z_{1}\right)$.

Property 1 ) says that $\|\cdot\|_{a d}$ is well defined. Properties 2 ) and 3 ) say that $\|\cdot\|_{a d}$ is a genuine metric on $\left.O(-Y)\right|_{Y}$. And property 4) says that (4.5) is an isomorphism.

Property 1) can be seen as follows: Assume $t \sim t^{\prime}$. Then $t=t^{\prime}+z_{1}^{2} F$ where $F$ is holomorphic on $U$, and $Y$ is defined by $z_{1}=0$. Then $\partial t=$ $\partial t^{\prime}+O\left(z_{1}\right)$, so $\left.\partial t\right|_{Y}=\left.\partial t^{\prime}\right|_{Y}$. Alternatively, property 1) follows from (4.6) since if $t=z_{1} F$ and $t^{\prime}=z_{1} F^{\prime}$ then $t \sim t^{\prime}$ if and only if $F-F^{\prime}$ is divisible by $z_{1}$. Since $z_{1}$ vanishes at $y$, property 1 ) follows.

To prove 2$)$, we note that in calculating $d(f t)$, the derivatives can land on $t$ or $f$. But when we restrict to $Y, t$ vanishes. Thus, for the purposes 
of calculating $\|f t\|_{a d}^{2}$, both derivatives land on $t$ and $|f|^{2}$ factors out. Alternatively, property 2) follows immediately from (4.6): If $t=z_{1} F$ then $f t=z_{1} \tilde{f} F$, where $\tilde{f}$ is any holomorphic extension of $f$ from $W \subseteq Y$ to $U \subseteq X$.

To prove 3), we work in local coordinates: If $t=z_{1} F$, where $F$ is a holomorphic function on $U,\|t\|_{a d}=|F(y)|^{2}$. On the other hand, $t$ vanishes at $y$ if and only if $F(y)=0$. This proves 3$)$.

Finally, 4) follows immediately from (4.6), and Theorem 4 is proved.

Remark: We also have the following relative version of Theorem 4: Let $S$ be a complex manifold and $\pi: \mathcal{X} \rightarrow S$ a smooth family of complex manifolds of relative dimension $n$. Let $\omega$ be a $(1,1)$ form on $\mathcal{X}$ such that the restriction of $\omega$ to each fiber $\mathcal{X}_{s}$ is a Kähler form. Let $\mathcal{Y} \subseteq \mathcal{X}$ be a smooth submanifold of codimension one such that $\mathcal{Y}_{s}=\mathcal{Y} \cap \mathcal{X}_{s}$ is smooth of codimension one. Let $\mathcal{K}_{\mathcal{Y}}$ and $\mathcal{K}_{\mathcal{X}}$ be the relative canonical bundles of $\mathcal{Y} \rightarrow S$ and $\mathcal{X} \rightarrow S$, and endow $\mathcal{K}_{\mathcal{X}}^{-1}$ with the metric $\omega^{n}$. Let $t$ be a section of $O(-\mathcal{Y}) \mid \mathcal{Y}$ over some open set $W \subseteq \mathcal{Y}$. Thus, as before, $t$ is represented by a holomorphic function $T$ on $U \subseteq \mathcal{X}$, with $U \cap \mathcal{X}=W$, with the property: $T$ vanishes on $\mathcal{Y}$. Define $\|t\|_{a d}^{2}$ by (4.4). Then $\left.K_{\mathcal{Y}} \otimes O(-\mathcal{Y})\right|_{\mathcal{Y}}=\left.\left(K_{\mathcal{X}}\right)\right|_{\mathcal{Y}}$ is an isometry with respect to this metric.

\subsection{The Aubin-Yau functional as a norm.}

The Aubin-Yau functional $\mathrm{AY}_{\omega}(\phi)$ on the space $P(M, \omega)$ of Kähler potentials was defined in subsection $§ 2.3$. In this subsection, we shall show that, for complete intersections, it can be written explicitly in terms of a norm on the space of defining polynomials. Let $(M, \omega)$ be a compact n-dimensional Kähler manifold, and $(L, h) \rightarrow M$ a holomorphic line bundle with metric $h$ satisfying $\operatorname{Ric}(h)=\omega$. The key property which we exploit is the close relation of $\mathrm{AY}_{\omega}(\phi)$ with the Deligne pairing

$$
\mathcal{A}_{h}=\langle L, \cdots, L\rangle^{\frac{1}{c_{1}(L)^{n}}}
$$

described in Theorem 2, which we reproduce here for convenience

$$
\mathcal{A}_{h e^{-\phi}}=\mathcal{A}_{h} \otimes O\left(\mathrm{AY}_{\omega}(\phi)\right)
$$

We wish to calculate $\mathcal{A}_{h}$ in the case where $M$ is a complete intersection. First we recall our previous notation: Let $N>n>0$ be positive integers, 
let $R_{d} \subseteq \mathbf{C}\left[z_{0}, \ldots, z_{N}\right]$ be the space of homogeneous polynomials of degree $d$, and let $Z=\mathbf{P}\left(R_{d_{1}}\right) \times \cdots \times \mathbf{P}\left(R_{d_{s}}\right)$ where $s=N-n$. Let

$$
\mathcal{X}=\left\{(z, x): z=\left(F_{1}, \ldots, F_{s}\right) \in Z, x \in \mathbf{P}^{N}, F_{1}(x)=\cdots=F_{s}(x)=0\right\}
$$

Then $\mathcal{X} \subseteq Z \times \mathbf{P}^{N}$ is a smooth variety and $\operatorname{dim}(\mathcal{X})=\operatorname{dim}(Z)+n$. Let $\mathcal{L} \rightarrow \mathcal{X}$ be the pull back of the hyperplane bundle on $\mathbf{P}^{N}$ equipped with the Fubini-Study metric. Let

$$
Z_{0}=\left\{z \in Z: \pi^{-1} z \quad \text { has dimension } n\right\}
$$

where $\pi: \mathcal{X} \rightarrow Z$ is the natural projection. Then $Z_{0} \subseteq Z$ is Zariski open and the restricted map $\pi: \mathcal{X}_{0} \rightarrow Z_{0}$ is flat (where $\mathcal{X}_{0}=\pi^{-1}\left(Z_{0}\right)$ ). Then the Deligne pairing

$$
\mathcal{S}=\langle\mathcal{L}, \ldots, \mathcal{L}\rangle\left(\mathcal{X}_{0} / \mathcal{Z}_{0}\right)
$$

is a line bundle on $Z_{0}$ with a continuous hermitian metric.

Let $H$ be the hyperplane bundle on the projective space $\mathbf{P}\left(R_{d}\right)$, endowed with the Fubini-Study metric. A global section of $H$ is a linear map $R_{d} \rightarrow \mathbf{C}$. Similarly, a global section of $\mathcal{L}^{d}$ is a polynomial map $\mathbf{C}^{N+1} \rightarrow \mathbf{C}$, homogeneous of degree $d$. And a global section of $H \otimes \mathcal{L}^{d}$ is a polynomial map $R_{d} \times \mathbf{C}^{N+1} \rightarrow \mathbf{C}$ which is homogeneous of degree one in the first variable, and degree $d$ in the second variable. As before, we observe that $H \otimes \mathcal{L}^{d} \equiv H \mathcal{L}^{d}$, which is a line bundle on $\mathbf{P}\left(R_{d}\right) \times \mathbf{P}^{N}$, has a canonical global section $\sigma_{d}$ given by the map $(F, z) \rightarrow f(z)$. The norm of this section is

$$
\left\|\sigma_{d}\right\|=\frac{|F(z)|}{|F| \cdot|z|^{d}}
$$

where $|F(z)|$ and $|z|$ are the norms of vectors in $\mathbf{C}^{s}$ and in $\mathbf{C}^{n+1}$ respectively, and $|F|$ is the norm of $\left(F_{1}, \cdots, F_{s}\right)$, viewed as an element of $R_{d_{1}} \times \cdots \times R_{d_{s}}$. We also observe that the curvature $c_{1}^{\prime}\left(H \mathcal{L}^{d}\right)$ is given by $c_{1}^{\prime}(H)+d c_{1}^{\prime}(\mathcal{L})$.

Now we consider the bundle

$$
\Gamma=\left\langle H_{1} \mathcal{L}^{d_{1}}, \ldots, H_{s} \mathcal{L}^{d_{s}}, \mathcal{L}, \ldots, \mathcal{L}\right\rangle\left(\left(Z_{0} \times \mathbf{P}^{N}\right) / Z_{0}\right)
$$

We evaluate $\Gamma$ in two different ways: First we use the multi-linearity of the Deligne pairing to write $\Gamma$ as a product of various terms: There is one term that involves none of the $H_{k}$ : As in the proof of Theorem 2, this term gives the trivial line bundle over $Z_{0}$ equipped with a constant metric. The terms that involve two $H_{k}$ are also trivial, by $(2.13)$. Thus

$$
\Gamma=\prod_{k=1}^{s}\left\langle\mathcal{L}^{d_{1}}, \ldots, H_{k}, \ldots, \mathcal{L}^{d_{s}}\right\rangle
$$


But now, applying (2.12), we get

$$
\Gamma=\prod_{k=1}^{s} H_{k}^{p_{k}}
$$

where $p_{k}=\prod_{j \neq k} d_{j}$.

On the other hand, we can evaluate $\Gamma$ in a different way, applying (2.6) $s$ times, using $\sigma_{1}, \ldots \sigma_{s}$ successively:

$$
\Gamma=\langle\mathcal{L}, \ldots, \mathcal{L}\rangle \otimes O(-E)
$$

where $E$ is the function on $Z_{0}$ defined by

$E\left(F_{1}, \ldots, F_{s}\right)=\frac{1}{2} \sum_{k=1}^{s} \int_{X_{k-1}} \log \left(\frac{\left|F_{k}(z)\right|^{2}}{\left|F_{k}\right|^{2} \cdot|z|^{2 d_{k}}}\right) \cdot\left(d_{k+1} \cdots d_{s}\right) \cdot c_{1}^{\prime}(\mathcal{L})^{N-k+1}$ and $X_{k}=\left\{z \in \mathbf{P}^{N}: F_{1}(z)=\cdots=F_{k}(z)=0\right\}$. Since $V_{k}=\int_{X_{k}} c_{1}^{\prime}(\mathcal{L})^{N-k}=$ $\left(d_{1} \cdots d_{k}\right)$ we conclude that $\langle\mathcal{L}, \ldots, \mathcal{L}\rangle$ is the line bundle $\prod_{k=1}^{s} H_{k}^{p_{k}}$ equipped with the metric

$$
\log \|F\|_{\mathcal{A}}^{2}=\sum_{k=1}^{s} p_{k} \cdot \frac{1}{V_{k}} \int_{X_{k}} \log \left(\frac{\left|F_{k}(z)\right|^{2}}{|z|^{2 d_{k}}}\right) \cdot c_{1}^{\prime}(\mathcal{L})^{N-k+1}
$$

where $F=\left(F_{1}, \ldots, F_{s}\right)$. Note that if we replace $F_{k}$ by $\lambda F_{k}$, then the right side is transformed by adding $p_{k} \log \lambda$ (which shows that the right side does indeed define a norm on $\left.\prod_{k=1}^{s} H_{k}^{p_{k}}\right)$.

We thus obtain the following:

Theorem 5. Let $M \subseteq \mathbf{P}^{N}$ be a complete intersection defined by the equations $F=0$, where $F=\left(F_{1}, \ldots, F_{s}\right)$ and $F_{k}$ is a homogeneous polynomial of degree $d_{k}$. Let $\omega$ be the Fubini-Study metric on $M$. Then for all $\sigma \in G L(N+1)$ we have

$$
\mathrm{AY}_{\omega}\left(\phi_{\sigma}\right)=\frac{1}{[\omega]^{n}} \log \frac{\left\|F^{\sigma}\right\|_{\mathcal{A}}^{2}}{\|F\|_{\mathcal{A}}^{2}}
$$

where $\phi_{\sigma}=\log \frac{|\sigma x|^{2}}{|x|^{2}}$ and $\|\cdot\|_{\mathcal{A}}$ is the norm on $\prod_{k=1}^{s} H_{k}^{p_{k}}$ defined by (4.8). 


\subsection{The Mabuchi K-energy as a degenerate norm.}

In this section we show that the Mabuchi $K$-energy of a complete intersection can be expressed in terms of a certain degenerate norm on the space of defining polynomials for $M$. This may be viewed as a non-linear generalization of Lu's formula. It also generalizes expressions for the $K$-energy in [T94] and in [PS1].

The Mabuchi $K$-energy was defined in $\S 2.3$. With the same notation as in there and in $\S 4.2$, we recall that it is closely related with the following Deligne pairing

$$
\mathcal{M}_{h}=\langle K, L, \ldots, L\rangle^{\frac{1}{c_{1}(L)^{n}}}\langle L, \ldots, L\rangle^{\frac{n}{n+1} \frac{c_{1}(M) c_{1}(L)^{n-1}}{\left[c_{1}(L)^{n}\right]^{2}}}
$$

where $K$ is endowed with the metric $\omega^{-n}$. More precisely, as stated in Theorem 2, we have

$$
\mathcal{M}_{h e^{-\phi}}=\mathcal{M}_{h} \otimes O\left(\nu_{\omega}(\phi)\right)
$$

Next consider the family $\mathcal{X}_{0} \rightarrow Z_{0} \subseteq Z_{0} \times \mathbf{P}^{N}$, where $Z_{0}=\{F \in Z: F=$ 0 is a normal variety of dimension $n\}$. : As before, let $\mathcal{L} \rightarrow \mathcal{X}_{0}$ be the pull back of the hyperplane bundle on $\mathbf{P}^{N}$ to the manifold $\mathcal{X}_{0}$, and let $\mathcal{K}^{-1} \rightarrow \mathcal{X}_{0}$ be the relative anti-canonical bundle, endowed with the metric $\omega_{\mathbf{P}^{N}}^{n}$. Now let

$$
\mathcal{M}=\langle\mathcal{K}, \mathcal{L}, \ldots, \mathcal{L}\rangle^{\frac{1}{c_{1}(L)^{n}}}\langle\mathcal{L}, \ldots, \mathcal{L}\rangle^{\frac{n}{n+1} \frac{c_{1}(M) c_{1}(L)^{n-1}}{\left[c_{1}(L)^{n}\right]^{2}}}
$$

Let $F \in Z_{0}$ and let $f \in \mathcal{M}_{F}$, where $\mathcal{M}_{F}$ is the fiber of $\mathcal{M}$ above $F$. The change of metrics formula (4.11) implies, for all $\sigma \in G L(N+1)$

$$
\nu_{\omega}\left(\phi_{\sigma}\right)=\log \frac{\left\|f^{\sigma}\right\|_{\mathcal{M}}^{2}}{\|f\|_{\mathcal{M}}^{2}}
$$

We wish to calculate the norm $\|\cdot\|_{\mathcal{M}}$. The adjunction formula says

$$
\mathcal{K}^{-1}=\mathcal{L}^{N+1} O\left(-Y_{1}\right)_{a d} \cdots O\left(-Y_{s}\right)_{a d}
$$

where $Y_{i} \subseteq \mathcal{X}_{0}$ is the hypersurface defined by $F_{i}(z)=0$ and $O\left(-Y_{i}\right)_{a d}$ is the bundle $O\left(-Y_{i}\right)$ endowed with the metric of Theorem 4. Here we are using the fact that

$$
K_{\mathbf{P}^{N}}^{-1}=\mathcal{O}_{\mathbf{P}^{N}}(N+1)
$$

Note that the isometry in (4.14) is equivariant with respect to the action of $S L(N+1)$, and not with respect to the action of $G L(N+1)$. It is for 
this reason that, unlike the transformation formula (4.8) for the Aubin-Yau functional, which holds for all $\sigma \in G L(N+1)$, our transformation formula for the K-energy will only hold for $\sigma \in S L(N+1)$.

Since $F_{i}(z)$ is a global section of $\mathcal{L}^{d_{i}} H_{i}$, we have a canonical isomorphism $\left(\mathcal{L}^{d_{i}} H_{i}\right)_{a d} \approx O\left(-Y_{i}\right)_{a d}$ for some uniquely defined metric $\|\cdot\|_{a d}$ on $\left(\mathcal{L}^{d_{i}} H_{i}\right)$. Thus $\mathcal{K}^{-1}=\mathcal{L}^{N+1}\left(\mathcal{L}^{d_{1}} H_{1}\right)_{a d}^{-1} \cdots\left(\mathcal{L}^{d_{s}} H_{s}\right)_{a d}^{-1}$. On the other hand, (4.4) implies that

$$
\left(\mathcal{L}^{d} H\right)_{a d}^{-1}=\left(\mathcal{L}^{d} H\right)^{-1} \otimes O\left(-\frac{1}{2} \log \left(\frac{\omega^{n-1} \wedge \frac{\sqrt{-1}}{2 \pi} \partial \bar{\partial} \frac{|F(z)|^{2}}{|z|^{2 d}|F|^{2}}}{\omega^{n}}\right)\right)
$$

Thus

$$
\left\langle\mathcal{K}^{-1}, \mathcal{L}, \ldots, \mathcal{L}\right\rangle=\left\langle\mathcal{L}^{N+1}\left(\mathcal{L}^{d_{1}} H_{1}\right)^{-1} \cdots\left(\mathcal{L}^{d_{s}} H_{s}\right)^{-1}, \mathcal{L}, \ldots, \mathcal{L}\right\rangle \otimes O(-G)
$$

where $G$ is the function on $\mathbf{P}\left(R_{d_{1}}\right) \times \cdots \times \mathbf{P}\left(R_{d_{s}}\right)$ given by

$$
G(F)=G\left(F_{1}, \ldots, F_{s}\right)=\frac{1}{2} \sum_{i=1}^{s} \int_{M} \log \left(\frac{\omega^{n-1} \wedge \frac{\sqrt{-1}}{2 \pi} \partial \bar{\partial} \frac{\left|F_{i}(z)\right|^{2}}{|z|^{2 d_{i}\left|F_{i}\right|^{2}}}}{\omega^{n}}\right) \cdot \omega^{n}
$$

Here $M$ is the variety $F=0$ and $\omega=\omega_{F S}$ is the Fubini-Study metric on $\mathbf{P}^{N}$. Let

$$
q_{k}=1-\frac{m}{d_{k}(n+1)}
$$

where $m=N+1-d_{1}-\cdots-d_{s}$. One sees easily that $q_{k} \geq 0$ with equality if and only if $d_{i}=1$ for all $i$. Then (4.15) implies

$$
\mathcal{M}=\prod_{i=0}^{s} H_{i}^{q_{i}}
$$

equipped with the singular metric

$$
\begin{aligned}
\log \|F\|_{\mathcal{M}}^{2}= & -\frac{m}{(n+1) d} \cdot \sum_{k=1}^{s} p_{k} \cdot \frac{1}{V_{k}} \int_{X_{k}} \log \left(\frac{\left|F_{k}(z)\right|^{2}}{|z|^{2 d_{k}}}\right) \cdot c_{1}^{\prime}(\mathcal{L})^{N-k+1} \\
& +\sum_{i=1}^{s} \int_{M} \log \left(\frac{\omega^{n-1} \wedge \frac{\sqrt{-1}}{2 \pi} \partial \bar{\partial} \frac{\left|F_{i}(z)\right|^{2}}{|z|^{2 d_{i}}}}{\omega^{n}}\right) \cdot \omega^{n}
\end{aligned}
$$

where $F=\left(F_{1}, \ldots, F_{s}\right)$. Applying (4.12) we deduce the following : 
Theorem 6. Let $M \subseteq \mathbf{P}^{N}$ be a smooth complete intersection defined by $F_{1}=\cdots=F_{s}=0$, where $F_{j}$ is homogeneous of degree $d_{j}$. For $\sigma \in S L(N+$ 1) let $\phi_{\sigma}=\log \frac{|\sigma(z)|^{2}}{|z|^{2}}$, with $z \in \mathbf{P}^{N}$. Let $d=d_{1} \cdots d_{s}$ and $p_{k}=d / d_{k}$. Let $\|F\|_{\mathcal{M}}$ be the degenerate metric defined by (4.16). Then for all $\sigma \in S L(N+1)$ we have

$$
\nu_{\omega}\left(\phi_{\sigma}\right)=\log \frac{\left\|F^{\sigma}\right\|_{\mathcal{M}}^{2}}{\|F\|_{\mathcal{M}}^{2}}
$$

Remark. The Aubin-Yau functional and the Mabuchi energy functional were written respectively in [Z] and [PS1] in terms of suitable norms of the Chow point of $M$. When $M$ is a hypersurface, the Chow point of $M$ coincides with $M$, and the norms $\|\cdot\|_{\mathcal{A}}$ and $\|\cdot\|_{\mathcal{M}}$ coincide with the norms $\|\cdot\|$ in $[\mathrm{Z}]$ and $\|\cdot\|_{\#}$ in [PS1]. Of course, when $M$ has higher codimension, no such direct comparison is possible, since the space of Chow points and the space of defining polynomials are then quite different. An exact expression for the Mabuchi functional on hypersurfaces and an asymptotic expression in the general case in terms of Quillen metrics can be found in [T94] and [T97]. See also $[\mathrm{P}]$ for an asymptotic expression for the Aubin-Yau functional.

Remark. The approach in the present paper can be extended to the case of irreducible normal projective varieties. This together with applications to stability will be reported elsewhere.

Dedication. This paper is dedicated to Professor Peter Li, former editorin-chief of the Communications in Analysis and Geometry, on the occasion of his fiftieth birthday.

\section{References.}

[A78] Aubin, T. Equations du type Monge-Ampère sur les varietes Kähleriennes compactes., Bull. Sc. Math. 102 (1978), 63-95

[EG] Edidin D. and Graham, W. Equivariant intersection theory, Invent. Math. 131 (1998), 595-634

[De] Deligne, P., Le determinant de la cohomologie, Contemp. Math. 67 (1987) 93-177.

[Do] Donaldson, S., Infinite determinants, stable bundles, and curvature, Duke Math. J. 54 (1987) 231-247. 
[DT] Ding, W. and Tian, G. Kähler-Einstein metrics and the generalized Futaki invariant, Invent. Math. 110 (1992), 315-335

[F83] Futaki, A. An obstruction to the existence of Einstein Kähler metrics, Invent. Math. 73 (1983), 437-443

[F87] Futaki, A. On a character of the automorphism group of a complact complex manifold, Invent. Math. 87 (1987), 655-660

[FMS] Futaki, A., Mabuchi, T., and Sakane, S. Einstein-Kähler metrics with positive Ricci curvature, Kähler metric and moduli spaces, 11-83, Adv. Stud. Pure Math., 18-II, Academic Press, Boston, MA, 1990.

[F-Mo] Futaki, A. and Morita, S. Invariant polynomials of the automorphism group of a complact complex manifold, J. Diff. Geom. 21 (1985), 135142

[Lu99] Lu, Z. On the Futaki invariants of complete intersections, Duke Math. J. 100 (1999), no. 2, 359-372.

[Lu01] Lu, Z. $K$ energy and $K$ stability on hypersurfaces, preprint: arXiv: math.DG/0108009.

[Ma] Mabuchi, T. K-energy maps integrating Futaki invariants, Tohoku Math. J., 38 (1986), 575-593

[P] Paul, S., Geometric analysis of Chow Mumford stability, Princeton Ph.D. thesis, 2000.

[PS1] Phong, D.H. and Sturm, J. Stability, energy functionals and KählerEinstein metrics, Comm. in Analysis and Geom., vol. 11 (2003), 563597, arXiv: math.DG/0203254

[PS2] Phong, D.H. and Sturm, J. Scalar curvature, moment maps and the Deligne Pairing, arXiv: math.DG/0209098, to appear in Amer. J. Math.

[T94] Tian, G. The K-energy on hypersurfaces and stability, Comm. in Analysis and Geom., vol. 2 (1994),239-265

[T97] Tian, G. Kähler-Einstein metrics with positive scalar curvature, Inv. Math., 137 (1994),1-37

[T00] Tian, G., Bott-Chern forms and geometric stability, Discrete Contin. Dynam. Systems 6 (2000) 211-220. 
[Y78] Yau, S.T. On the Ricci curvature of a compact Kähler manifold and the comples Monge-Ampère equation I, Comm. Pure Appl. Math 31 (1978) 339-411

[Y93] Yau, S.T. Open problems in geometry, Proc. Sym. Pure Math. 54 (1993) 1-28

[Yo98] Yotov, M. Generalized Futaki invariant of almost Fano Toric Varieties, Examples, arXiv: math.AG/9806116

[Yo99] Yotov, M. On the generalized Futaki invariant, arXiv: math.AG / 9907055

[Z] Zhang, S. Heights and reductions of semi-stable varieties, Comp. Math. 104 (1996), 77-105

D. H. PHONG

Department of Mathematics

Columbia University

New YoRK, NY 10027

phong@math.columbia.edu

JACOB STURM

Department of Mathematics

RUTGERS UNIVERSITY

NEWARK, NJ 07102

sturm@andromeda.rutgers.edu 
D. H. Phong and J. Sturm 